\title{
QUOTIENT DE HADAMARD EN PLUSIEURS VARIABLES
}

\author{
ABDelKader NECER
}

We obtain some results about a conjecture of Hadamard's quotient of rational series in the case of several variables.

\section{INTRODUCTION}

Soit $K$ un corps commutatif de caractéristique nulle, $f$ et $g$ deux séries formelles à coefficients dans $K$. Le quotient de Hadamard, quand il existe, de $f$ par $g$ est la série, disons $h$, dont les coefficients sont les quotients (terme à terme) des coefficients de $f$ et $g$.

En réponse à une conjecture de Pisot et pour courronner les travaux de Cantor, Pourchet, Bézivin et tant d'autres auteurs, Van der Poorten a montré en 1986 (voir [5]) que, si on suppose que $f$ et $g$ sont des séries rationnelles en une variable et si les coefficients de la série quotient $h$ sont dans un anneau de type fini sur $\mathbb{Z}$, alors la série $h$ est rationnelle.

Dans ce papier on s'intéresse à l'analogue en plusieurs variables de cette conjecture (devenue donc le théorème de Van der Poorten). En utilisant à la fois ce dernier théorème et un résultat dû à Cantor, on donne deux réponses partielles à cette question. La première (voir Proposition 1) concerne le cas où $f$ est rationnelle et $g$ a pour coefficients des produits de suites récurrentes linéaires. La seconde réponse traite du cas où $f$ est une série "reconnaissable" et $g$ est une série reconnaissable particulière, ses coefficients sont des polynômes (voir Proposition 2).

Dans une autre direction, on généralise au cas de plusieurs variables, le théorème de Bézivin de la version approchée de la conjecture de Pisot : si les coefficients de la série $h$ sont décomposés en une somme d'éléments entiers et d'éléments dont la croissance est géométrique, alors la série dont les coefficients sont entiers est rationnelle.

Même si les résultats restent valables quelque soit le nombre de variables, pour plus de clarté et par souci d'alléger l'écriture, on ne traitera ici que le cas de deux variables.

Received 19th November, 1996

Que J.P. Bézivin trouve ici l'expression de mes remerciements pour son aide et ses précieux conseils. Mes remerciements vont aussi à toute l'équipe du Séminaire d'Analyse Ultramétrique de Paris VI pour leur accueil et leur disponibilité.

Copyright Clearance Centre, Inc. Serial-fee code: 0004-9729/97 \$A2.00+0.00. 


\section{DÉfinitions et NOTATIONS}

$\mathrm{K}$ désignera un corps commutatif, algébriquement clos et de caractéristique nulle et $A$ un sous anneau de $K$ de type fini sur $\mathbb{Z}$.

2.1. DÉfinition Soit

$$
f(x, y)=\sum_{(m, n) \in \mathbb{N}^{2}} a(m, n) x^{m} y^{n} \in K[[x, y]]
$$

On dit que $f$ est une série rationnelle s'il existe $p$ et $q$ deux éléments de $K[x, y]$ tels que

$$
q \neq 0 \quad \text { et } \quad q f=p \text {. }
$$

L'ensemble des séries rationnelles est noté $R(K)$.

\subsection{DÉfinition Soit}

$$
f(x, y)=\sum_{(m, n) \in \mathbb{N}^{2}} a(m, n) x^{m} y^{n} \quad \text { et } \quad g(x, y)=\sum_{(m, n) \in \mathbb{N}^{2}} b(m, n) x^{m} y^{n}
$$

deux éléments de $K[[x, y]]$. Le produit de Hadamard de $f$ et $g$ est la série, notée $f * g$, et donnée par:

$$
(f * g)(x, y)=\sum_{(m, n) \in \mathbb{N}^{2}} a(m, n) b(m, n) x^{m} y^{n} .
$$

2.3. Remarque Contrairement au cas d'une variable $R(K)$ n'est pas stable pour le produit de Hadamard comme on peut le voir sur l'exemple suivant:

$$
f(x, y)=\sum_{(m, n) \in \mathbb{N}^{2}}\left(\begin{array}{c}
m+n \\
n
\end{array}\right) x^{m} y^{n}=\frac{1}{1-x-y} \in R(K)
$$

mais la série

$$
(f * f)(x, y)=\left((1-x-y)^{2}-4 x y\right)^{-1 / 2}
$$

n'appartient pas à $R(K)$.

2.4. Définition Soit $f(x, y)=\sum_{(m, n) \in \mathbb{N}^{2}} a(m, n) x^{m} y^{n}$. On dit que $f$ est une série reconnaissable s'il existe $t \in \mathbb{N}^{*}$, des polynômes $P_{1}, \ldots, P_{t}$ dans $K[x, y]$ et des éléments $\alpha_{1}, \ldots, \alpha_{t} ; \beta_{1}, \ldots, \beta_{t}$ dans $K$ tels que

$$
a(m, n)=\sum_{j=1}^{t} P_{j}(m, n) \alpha_{j}^{m} \beta_{j}^{n} \quad \forall(m, n) \in \mathbb{N}^{2} .
$$

L'ensemble des séries reconnaissables est noté $R_{0}(K)$. On a $R_{0}(K) \subset R(K)$ et $\left(R_{0}(K)\right.$,*) est une $K$-algèbre. 
2.5. Conjecture Soit

$$
f(x, y)=\sum_{(m, n) \in \mathbb{N}^{2}} a(m, n) x^{m} y^{n} \quad \text { et } \quad g(x, y)=\sum_{(m, n) \in \mathbb{N}^{2}} b(m, n) x^{m} y^{n}
$$

deux éléments de $R(K)$.

On suppose que

$$
b(m, n) \neq 0 \quad \text { et } \quad c(m, n)=\frac{a(m, n)}{b(m, n)} \in A \quad \forall(m, n) \in \mathbb{N}^{2} .
$$

Alors

$$
h(x, y)=\sum_{(m, n) \in \mathbb{N}^{2}} c(m, n) x^{m} y^{n} \in R(K)
$$

2.6. Remarques

(a) Il y'a bien entendu des quotients de Hadamard de séries rationnelles qui ne le sont pas comme on peut le voir sur l'exemple suivant : la série

$$
\sum_{m \geqslant 1, n \geqslant 1} \frac{1}{m n} x^{m} y^{n} \notin R(K),
$$

mais est quotient des séries rationnelles

$$
\sum_{m \geqslant 1, n \geqslant 1} x^{m} y^{n}=\frac{x y}{(1-x)(1-y)}, \sum_{m \geqslant 1, n \geqslant 1} m n x^{m} y^{n}=\frac{x y}{(1-x)^{2}(1-y)^{2}} .
$$

(b) L'hypothèse (h) est nécessaire comme on peut le voir en utilisant les récurrences qui découlent de la relation $(R)$.

\section{ENONCÉS DES RÉSULTATS ET RAPPELS}

Un cas particulier de la conjecture est donné par la

Proposition 1. Soit

$$
f(x, y)=\sum_{(m, n) \in \mathbb{N}^{2}} a(m, n) x^{m} y^{n}, g(t)=\sum_{n \geqslant 0} b(n) t^{n} \quad \text { et } \quad h(t)=\sum_{n \geqslant 0} c(n) t^{n}
$$

des séries rationnelles à coefficients dans $K$.

On suppose que

$$
b(n) c(m) \neq 0 \quad ; \quad \frac{a(m, n)}{b(n) c(m)} \in A \quad \forall(m, n) \in \mathbb{N}^{2} .
$$

Alors

$$
\sum_{m, n} \frac{a(m, n)}{b(n) c(m)} x^{m} y^{n} \in R(K)
$$


Le résultat reste vrai si on prend pour $(b(n))_{n \in \mathbb{N}}$ et $(c(n))_{n \in \mathbb{N}}$ des suites particulières, par exemple des polynômes en $n$.

Un autre cas particulier de la conjecture est donné par la

Proposition 2. Soit

$$
f(x, y)=\sum_{(m, n) \in \mathbb{N}^{2}} a(m, n) x^{m} y^{n} \in K[[x, y]] \quad \text { et } P(x, y) \in K[x, y]
$$

On suppose qu'il existe

$$
t \in \mathbb{N}^{*},\left(\alpha_{1}, \beta_{1}\right), \ldots,\left(\alpha_{t}, \beta_{t}\right) \quad \text { dans } \quad K^{2} \quad \text { et } P_{1}, \ldots, P_{t} \quad \text { dans } K[x, y]
$$

tels que

(1) $\quad a(m, n)=\sum_{j=1}^{t} P_{j}(m, n) \alpha_{j}^{n} \beta_{j}^{m} \quad \forall(m, n) \in \mathbb{N}^{2}$,

(2) $P(m, n) \neq 0 \quad$ et $h(m, n)=a(m, n) / P(m, n) \in A \quad \forall(m, n) \in \mathbb{N}^{2}$.

Alors

$$
T(x, y)=\sum_{(m, n) \in \mathbb{N}^{2}} h(m, n) x^{m} y^{n} \in R(K) .
$$

Pour la démonstration de ces deux propositions on aura besoin des résultats suivants :

ThÉorème 1. (Quotient de Hadamard - Van der Poorten [5].)

Soit

$$
f(x)=\sum_{n \geqslant 0} a(n) x^{n} \quad \text { et } \quad g(x)=\sum_{n \geqslant 0} b(n) x^{n} \quad \text { dans } \quad R(K) .
$$

On suppose que

$$
b(n) \neq 0 \quad \text { et } \quad c(n)=\frac{a(n)}{b(n)} \in A \quad \text { pour tout } \quad n \in \mathbb{N} .
$$

Alors la série $\sum_{n \geqslant 0} c(n) x^{n}$ est dans $R(K)$.

3.1. Remarque Ce théorème, démontré par Van der Poorten en 1986, constitue une réponse à la conjecture de Pisot (voir [4] pour une rédaction de la démonstration). La preuve de ce résultat suit le schéma donné par Pourchet [3] et fait notamment appel, dans le cas algébrique, au théorème 2 ci-dessous. En utilisant de nouveau le théorème de Van der Poorten, on obtient une version en caractéristique nulle, du théorème de Cantor.

ThÉorème 2. (Cantor [2].) Soit

$$
f(x)=\sum_{n \geqslant 0} a(n) x^{n} \quad \text { dans } \quad R(K) \quad \text { et } \quad P(x) \quad \text { dans } K[x] .
$$

On suppose que

$$
P(n) \neq 0 \quad \text { et } \quad \frac{a(n)}{P(n)} \in A \text { pour tout } n \geqslant 0
$$


Alors

De plus si

$$
\sum_{n \geqslant 0} \frac{a(n)}{P(n)} x^{n} \in R(K)
$$

$$
a(n)=\sum_{i=1}^{t} P_{i}(n) \alpha_{i}^{n},
$$

où $\alpha_{1}, \ldots, \alpha_{t}$ sont les fréquences de $f$ dans $K$ et $P_{1}, \ldots, P_{t}$ des polynômes, alors

$$
P(x) \text { divise } P_{i}(x) \text { dans } K[x] \text { pour tout } i=1, \ldots, t .
$$

Une version "approchée" du théorème 1 a été obtenue par Bézivin dans [1]. Elle se généralise au cas de plusieurs variables. Ici $K$ est le corps des nombres complexes.

Proposition 3. Soit

$$
g(x, y)=\sum_{(m, n) \in \mathbb{N}^{2}} b(m, n) x^{m} y^{n} \in R_{0}(K) .
$$

Pour tout $(m, n) \in \mathbb{N}^{2}, b(m, n)$ est donc de la forme (1). On suppose que

(1) $\left|\alpha_{1}\right|>\left|\alpha_{i}\right|, \quad\left|\beta_{1}\right|>\left|\beta_{i}\right| \quad \forall i \geqslant 2$,

(2) $\quad P_{1}(x, y)=\lambda_{1} \quad$ est une constante non nulle.

Soit $f(x, y)=\sum_{(m, n) \in \mathbb{N}^{2}} a(m, n) x^{m} y^{n} \in R(K)$, on suppose qu'il existe $\rho \in\left[\begin{array}{ll}0 & 1[\text { et }\end{array}\right.$ $\forall(m, n) \in N^{2}$, il existe $c(m, n) \in \mathbb{Z}$ et $d(m, n)=O(\rho)$ dans $K$ tels que

$$
b(m, n) \neq 0 \quad \text { et } \quad \frac{a(m, n)}{b(m, n)}=c(m, n)+d(m, n) .
$$

Alors

$$
\sum_{(m, n) \in \mathbb{N}^{2}} c(m, n) x^{m} y^{n} \in R(K)
$$

\section{Preuve de la proposition 1}

Soit $f(x, y)=\sum_{(m, n) \in \mathbb{N}^{n}} a(m, n) x^{m} y^{n}=p(x, y) / q(x, y)$ où $p$ et $q \neq 0$ dans $K[x, y]$.

(a) Quitte à agrandir $A$, on peut supposer que $p$ et $q$ sont dans $A[x, y]$.

(b) Soit

$$
h(m, n)=\frac{a(m, n)}{b(n) c(m)} \quad \forall(m, n) \in \mathbb{N}^{2} .
$$

Comme $h(m, n) \in A \quad \forall(m, n) \in \mathbb{N}^{2}$, les $c(m) h(m, n)$ sont encore dans un anneau de type fini sur $\mathbb{Z}$ car la série $\sum_{m \in \mathbb{N}} c(m) x^{m}$ est rationnelle. Il suffit donc de montrer que la proposition est vraie pour la série

$$
\sum_{(m, n) \in \mathbb{N}^{2}} \frac{a(m, n)}{b(n)} x^{m} y^{n}
$$

(c) La série $f(x, y)$ peut s'écrire 
On a alors

$$
f(x, y)=\sum_{n \geqslant 0} \varphi_{n}^{\prime}(y) x^{n} \quad \text { où } \quad \varphi_{n}^{\prime}(y)=\sum_{m \geqslant 0} a(m, n) y^{m}
$$

$$
\frac{\partial^{k} f(x, y)}{\partial x^{k}}=\sum_{n \geqslant k} n(n-1) \cdots(n-k+1) \varphi_{n}^{\prime}(y) x^{n-k} \quad \forall k \geqslant 0 .
$$

Dond

$$
\frac{\partial^{k} f(0, y)}{\partial x^{k}}=k ! \varphi_{k}^{\prime}(y)=k ! \sum_{m \geqslant 0} a(k, m) y^{m} \quad \forall k \geqslant 0
$$

Par conséquent

$$
\varphi_{k}^{\prime}(y)=\frac{1}{k !} \times \frac{\partial^{k} f(0, y)}{\partial x^{k}}=\sum_{m \geqslant 0} a(k, m) y^{m} \quad \forall k \geqslant 0,
$$

et donc $\varphi_{k}^{\prime}(y) \in R(K)$.

(d) Comme $f(x, y)=p(x, y) / q(x, y)$, en dérivant par rapport à $x$ et en raisonnant par récurrence sur $n$ on obtient

$$
\frac{1}{n !} \times \frac{\partial^{n} f(x, y)}{\partial x^{n}}=\frac{\psi_{n}(x, y)}{q(x, y)^{n+1}} \quad \text { avec } \quad \psi_{n}(x, y) \in K[x, y]
$$

(e) Soit

$$
h(x, y)=\sum_{n \geqslant 0, m \geqslant 0} \frac{a(m, n)}{b(n)} x^{m} y^{n} \quad \text { et } \quad \varphi_{n}(y)=\frac{\varphi_{n}^{\prime}(y)}{b(n)} \quad \forall n \geqslant 0 .
$$

On a alors grâce à (2) et (3) :

$$
\varphi_{n}(y)=\frac{\psi_{n}(0, y)}{b(n) q(0, y)^{n+1}} \quad \forall n \geqslant 0,
$$

ou encore :

$$
q(0, y)^{n+1} \varphi_{n}(y)=\frac{1}{b(n)} \psi_{n}(0, y) \quad \forall n \geqslant 0 .
$$

Comme $\psi_{n}(x, y) \in K[x, y], 1 /(b(n)) \psi_{n}(0, y) \in K[y]$, donc

$$
q(0, y)^{n+1} \varphi_{n}(y) \in K[y]
$$

Or, d'une part $q(0, y)^{n+1} \in A[y]$ et d'autre part, grâce à $(H), \varphi_{n}(y) \in A[[y]]$. Donc

$$
q(0, y)^{n+1} \varphi_{n}(y) \in A[y] .
$$

Sachant que $q(0, y)^{n+1} \in A[y]$, on a

$$
\varphi_{n}(y) \in A\left[y, q(0, y)^{-1}\right]=B \text {. }
$$

Avec $q(0, y) \neq 0$ car $q(0,0) \neq 0$ et B est bien entendu un anneau de type fini sur $\mathbb{Z}$. 
(f) On a donc

$$
f(x, y)=\sum_{n \geqslant 0} \varphi_{n}^{\prime}(y) x^{n} \in K(y)(x)
$$

et aussi

$$
\sum_{n \geqslant 0} b(n) x^{n} \in K(x) \subset K(y)(x) \quad \text { où } \quad \varphi_{n}(y)=\frac{\varphi_{n}^{\prime}(\dot{y})}{b(n)} \in B \quad \forall n \geqslant 0 .
$$

Le théorème 1, appliqué au corps $K(y)$ et son sous anneau $B$, permet alors d'écrire

$$
\sum_{(m, n) \in \mathbb{N}^{2}} h(m, n) x^{m} y^{n}=\sum_{n \geqslant 0} \varphi_{n}(y) x^{n} \in K(y)(x) .
$$

Ou encore

$$
\sum_{(m, n) \in \mathbb{N}^{2}} h(m, n) x^{m} y^{n} \in R(K) .
$$

\section{Preuve de la proposition 2}

Elle utilise la proposition 1 et le fait que si un polynôme, disons $P\left(x_{1}, \ldots, x_{s}\right)$, s'annule pour tout $\left(\alpha_{1}, \ldots, \alpha_{s}\right)$ dans $E_{1} \times \ldots \times E_{s}$ où, $\operatorname{Card}\left(E_{i}\right)>\operatorname{deg}_{x_{i}} P$ pour tout $i \in[1, \ldots, s]$, alors $P \equiv 0$.

(a) Soit $a(m, n)=\sum_{i=1}^{t} P_{i}(m, n) \alpha_{i}^{n} \beta_{i}^{m}$ et $h(m, n)=a(m, n) / P(m, n) \forall(m, n) \in \mathbb{N}^{2}$.

Soit $i \in[1,2, \ldots, t]$. La division Euclidienne de $P_{i}(x, y)$ par $P(x, y)$ dans $K(y)[x]$ donne

$$
(\exists B(y) \in A[y]): B(y) P_{i}(x, y)=P(x, y) S_{i}(x, y)+R_{i}(x, y)
$$

où

$$
S_{i}(x, y) \in K[x, y], R_{i}(x, y) \in K[x, y] \text { et } R_{i}(x, y)=0 \text { ou } \operatorname{deg}_{x} R_{i}<\operatorname{deg}_{x} P \text {. }
$$

(b) Il suffit de montrer que $R_{i}(x, y)=0$ pour $i=1,2, \ldots, t$.

Si c'est le cas on a

$$
B(m) P_{i}(m, n)=P(m, n) S_{i}(m, n) \forall 1 \leqslant i \leqslant t, \forall(m, n) \in \mathbb{N}^{2} .
$$

En posant : $t(m, n)=(B(m) a(m, n)) / P(m, n) \quad \forall(m, n) \in \mathbb{N}^{2}$, on obtient

$$
t(m, n)=\sum_{i=1}^{t} S_{i}(m, n) \alpha_{i}^{n} \beta_{i}^{m} \quad \forall(m, n) \in \mathbb{N}^{2} .
$$

Ce qui signifie

$$
\sum_{(m, n) \in \mathbb{N}^{2}} t(m, n) x^{m} y^{n} \in R_{0}(K) \subset R(K) .
$$

La proposition est alors une conséquence de la proposition 1 car

$$
\frac{a(m, n)}{P(m, n)}=\frac{t(m, n)}{B(m)} \quad \forall(m, n) \in \mathbb{N}^{2}
$$


Remarquons que

$$
B(m) \neq 0 \quad \forall m \in \mathbb{N} .
$$

Le contraire entrainerait une contradiction avec $\operatorname{deg}_{x} R_{i}<\operatorname{deg}_{x} P$ (il suffit de remplacer dans (4)).

(c) Montrons donc que $R_{i}(x, y)=0 \quad \forall 1 \leqslant i \leqslant t$.

Soit $i \in\{1,2, \ldots, t\}$ et soit $m \in \mathbb{N}$, l'hypothèse $h(m, n) \in A$ entraine, en utilisant le théorème 2 , que

$$
P(x, m) \text { divise } \beta_{i}^{m} P_{i}(x, m)
$$

Soit

$$
\beta_{i}^{m} P_{i}(x, m)=P(x, y) \varphi_{i, m}(x, m), \quad \varphi_{i, m}(x, m) \in K[x] .
$$

En remplaçant dans (4) on obtient

$$
\frac{B(m)}{\beta_{i}^{m}} P(x, m) \varphi_{i, m}(x)=P(x, m) S_{i}(x, m)+R_{i}(x, m) .
$$

C'est-à-dire,

$$
R_{i}(x, m)=P(x, m)\left[\frac{B(m)}{\beta_{i}^{m}} \varphi_{i, m}(x)-S_{i}(x, m)\right] .
$$

Comme $\operatorname{deg}_{x} R_{i}<\operatorname{deg}_{x} P$, il vient:

$$
R_{i}(x, m)=0 \quad \forall m \in \mathbb{N}
$$

Ce qui n'est possible que si $R_{i}(x, y)=0$.

On a donc $R_{i}(x, y)=0 \quad \forall i \in[1, \ldots, t]$. D' où la proposition.

\section{Preuve De la proposition 3}

(a) On a

$$
b(m, n)=\sum_{j=1}^{t} P_{j}(m, n) \alpha_{j}^{n} \beta_{j}^{m}, \quad P_{1}(m, n)=\lambda_{1} \neq 0 \quad \forall(m, n) \in \mathbb{N}^{2}
$$

et

$$
\left|\alpha_{1}\right|>\left|\alpha_{i}\right|,\left|\beta_{1}\right|>\left|\beta_{i}\right| \quad \forall i \geqslant 2
$$

(b) Pour $(m, n) \in \mathbb{N}^{2}, b(m, n)$ s'écrit:

$$
b(m, n)=\lambda_{1} \alpha_{1}^{n} \beta_{1}^{m}(1-R(m, n))
$$

où

$$
R(m, n)=-\sum_{j=1}^{t} \frac{P_{j}(m, n)}{\lambda_{1}}\left(\frac{\alpha_{j}}{\alpha_{1}}\right)^{n}\left(\frac{\beta_{j}}{\beta_{1}}\right)^{m}
$$


De (5) on tire

$$
R(m, n)=O\left(\varepsilon^{n+m}\right) . \quad \text { où } 0<\varepsilon<1 .
$$

(c) Pour tout $M \in \mathbb{N}$ on a

$$
\frac{a(m, n)}{b(m, n)}=\lambda_{1}^{-1} \alpha_{1}^{-n} \beta_{1}^{-m}\left(1+R(m, n)+\cdots+R(m, n)^{M}\right)+\frac{a(m, n)}{b(m, n)} R(m, n)^{M+1} .
$$

Par suite (6) donne

$$
R(m, n)^{M+1}=O\left(\varepsilon^{(n+m)(M+1)}\right) .
$$

(d) Comme il existe $B \in \mathbb{R}^{+}$tel que $a(m, n)=O\left(B^{n+m}\right)$ car

$$
\sum_{(m, n) \in \mathbb{N}^{2}} a(m, n) x^{n} y^{m} \quad \text { est rationnelle }
$$

et que

$$
b(m, n) \geqslant \frac{1}{2}\left|\lambda_{1}\right| \alpha_{1}^{n} \mid \beta_{1}^{m}
$$

il existe $A \in \mathbb{R}^{+}$tel que

$$
\frac{a(m, n)}{b(m, n)}=O\left(A^{n+m}\right) \quad \forall(m, n) \in \mathbb{N}^{2} .
$$

A partir de maintenant on choisit $M$ de telle manière que:

$$
\varepsilon^{M+1} A \leqslant \rho<1 .
$$

Ce qui donne

$$
\frac{a(m, n)}{b(m, n)} R(m, n)^{M+1}=O\left(\rho^{n+m}\right)
$$

(e) Posons

$$
H_{M}(m, n)=a(m, n) \lambda_{1}^{-1} \alpha_{1}^{-n} \beta_{1}^{-m}\left(1+R(m, n)+\cdots+R(m, n)^{M}\right)
$$

et

$$
S_{M}(m, n)=\frac{a(m, n)}{b(m, n)} R(m, n)^{M+1}
$$

On a donc

$$
\sum_{m, n} \frac{a(m, n)}{b(m, n)} x^{m} y^{n}=\sum_{m, n} H_{M}(m, n) x^{m} y^{n}+\sum_{m, n} S_{M}(m, n) x^{m} y^{n}
$$

avec

$$
H(x, y)=\sum_{m, n} H_{M}(m, n) x^{m} y^{n} \in R(K)
$$


car, comme le montre la remarque 1, produit d'une série rationnelle et d'une série reconnaissable. De plus d'après (7)

$$
S_{M}(m, n)=O\left(\rho^{n+m}\right)
$$

(f) Comme par hypothèse

$$
\frac{a(m, n)}{b(m, n)}=c(m, n)+d(m, n) \quad \forall(m, n) \in \mathbb{N}^{2},
$$

il vient

$$
c(m, n)+d(m, n)=H_{M}(m, n)+S(m, n) \quad \forall(m, n) \in \mathbb{N}^{2} .
$$

On peut donc écrire

$$
c(m, n)=H_{M}(m, n)+S^{\prime}(m, n) \quad \forall(m, n) \in \mathbb{N}^{2} .
$$

Où

$$
S^{\prime}(m, n)=H_{M}(m, n)-d(m, n) \quad \forall(m, n) \in \mathbb{N}^{2} .
$$

Comme $H(x, y) \in R(K)$, il existe $l \in \mathbb{N},\left(e_{1}, \ldots, e_{l}\right) \in \mathbb{Z}^{l} ; E$ un sous ensemble fini de $\mathbb{N}^{2}$ et $l$ éléments de $\mathbb{N}^{2}$, notés $\left(n_{i}, m_{i}\right)_{1 \leqslant i \leqslant l}$ tels que:

$$
\sum_{i=1}^{l} e_{i} H_{M}\left(n-n_{i}, m-m_{i}\right)=0 \quad \forall(m, n) \in \mathbb{N}^{2}-E .
$$

Ou encore en utilisant (9)

$$
\sum_{i=1}^{l} e_{i} c\left(n-n_{i}, m-m_{i}\right)=\sum_{i=1}^{l} e_{i} S_{M}^{\prime}\left(n-n_{i}, m-m_{i}\right) \quad \forall(m, n) \in \mathbb{N}^{2}-E .
$$

Posons

$$
X(m, n)=\sum_{i=1}^{l} e_{i} S_{M}^{\prime}\left(n-n_{i}, m-m_{i}\right) \quad \forall(m, n) \in \mathbb{N}^{2} .
$$

D'une part $X(m, n) \in \mathbb{Z}$ car les $e_{i}, 1 \leqslant i \leqslant l$, et les $c(m, n)$ sont entiers; d'autre part $O\left(\rho^{n+m}\right)$ (d'après l'hypothèse sur les $d(m, n)$ et l'équation $\left.(9)\right)$ donc

$$
X(m, n)=0 \quad \forall(m, n) \in \mathbb{N}^{2}-E^{\prime}
$$

où $E^{\prime}$ est un sous ensemble fini de $\mathbb{N}^{2}$. Par conséquent

$$
\sum_{i=1}^{l} e_{i} c\left(n-n_{i}, m-m_{i}\right)=0 \quad \forall(m, n) \in \mathbb{N}^{2}-E \cup E^{\prime}
$$

D'où la proposition. 


\section{BIBLIOGRAPHE}

[1] J.P. Bézivin, 'Indépendance linéaire des valeurs des solutions transcendantes de certaines équations fonctionnelles II', Acta Arith. 55 (1990), 233-290.

[2] D.J. Cantor, 'On arithmetic properties of the Taylor series of rational functions', Canad. J. Math. 21 (1969), 378-382.

[3] Y. Pourchet, 'Solution du problème arithmétique du quotient de Hadamard de deux fractions rationnelles', C.R. Acad Sci. Paris Sér. A 288 (1979), 1055-1057.

[4] R. Rumely, 'Notes on Van der Poorten's proof of the Hadamard quotient theorem I et II', Sém. Théor. Nombres Bordeaux (1986/87), 349-409.

[5] A.J. Van der Poorten, 'Solution de la conjecture de Pisot sur le quotient de Hadamard de deux fractions rationnelles', C.R. Acad. Sci. Paris. 306 (1988), 97-102.

DMI Université de Metz

Ile de Saulcy, BP 800794

57012 Metz cedex 01

France 